\title{
Grid Monte Carlo Simulation of a Medical Linear Accelerator
}

\author{
Samir Didi, Mustapha Zerfaoui, Abdelilah Moussa, Yassine Benkhouya, Mehdi el Ouartiti
}

\begin{abstract}
A full grid simulation of the head of an Elekta Synergy Platform medical linear accelerator is performed using the Geant4 Monte Carlo platform. The simulation includes all components of the accelerator head and a homogeneous water phantom. Results in terms of depth doses and lateral dose profiles are presented for $6 \mathrm{MV}$ photon beam with the $10 \times 10 \mathrm{~cm}^{2}$ reference field size at $100 \mathrm{~cm}$ distance from the source. Overall, a good agreement with the measured dose data is achieved with a precision better than $0.93 \%$ and $2.63 \%$ for the depth dose profile and lateral dose profiles respectively.
\end{abstract}

Index Terms_-Grid Computing; TPS; Linac; Radiotherapy; Geant4.

\section{INTRODUCTION}

It is well known that the Monte Carlo simulation provides the most accurate method for modeling the radiotherapy treatment. However, its widespread use is constrained by the high computational cost. The progress made with the grid computing could open a new window for the application of Monte Carlo simulation of radiotherapy treatments in the clinical environment as a normal routine. Indeed, grid computing facilities offer a cheap access to a distributable and reliable powerful computing capability and therefore a dramatic acceleration of the simulation could be reached for a complex system [1].

The success of Monte-Carlo modeling of a medical linear accelerator depends crucially on the information implemented on each component of the treatment head, namely the materials, geometric shapes, size and precise position of the various Linac components [17], [18].

In this work, we present the simulation of an Elekta Synergy Platform medical linear accelerator treatment head developed on the grid using the last public release v10.3 of Geant4 Monte Carlo platform. The goal is to calculate the dose distributions in a water phantom keeping the accuracy of results within 3\%. Geant4 (GEometry ANd Tracking) [2]-[6] is an advanced Opensoure toolkit developed by the international Geant4 collaboration for high energy, nuclear and accelerator physics as well as for medical and space science studies[23].

Among the several codes, Geant 4 is widely used for its modern technology, its flexibility and its impressive

Published on December 14, 2018

S. Didi is with the Regional Oncology Center of Meknes, Boulevard Moulay Youssef, Meknes, Morocco (e-mail: didisamir@gmail.com)

M. Zerfaoui and A. Moussa are with the LPMR, Faculty of Sciences, University Mohammed First, PO. Box 717, Oujda, 60000, Morocco (email: zerfaoui@yahoo.fr)

M. el Ouartiti and Y. Benkhouya are with the ESMAR, Faculty of Sciences, University of Mohammed V, BP. 1014, Rabat, Morocco. (e-mail: mehdielourtiti01@gmail.com) capabilities. Geant4 provides the users with tools to handle complex geometries with a set of very accurate physics lists [7], [8]. Tailored to multiple applications and available for accurate interaction and transport of many types of particle in the matter. In addition, Geant 4 offers interfaces to enable the users to interact easily with their application through several visualization drivers.

The paper is organized as follows: Section 2 describes the Geant 4 model and the simulation on the grid. In section 3 we present and discuss the results of our model for the reference field. Finally, section 4 presents our conclusion.

\section{MATERIALS AND METHODS}

\section{A. Simulation of the gantry}

Based on the vendor detailed information, we simulated the head of the linear accelerator, by use of Geant 4 platform. Fig. 1 illustrates the global head structure of the linear accelerator and the water phantom considered in this study. Simulated components include the:

- Target: is a disk of approximately $0.9 \mathrm{~mm}$ height made of the tungsten alloy target $(10 \% \mathrm{Re}, 90 \% \mathrm{~W}$, $19.49 \mathrm{~g} / \mathrm{cm}^{3}$ ), creates Bremsstrahlung X-rays, and the remaining primary electrons are absorbed in a graphite absorber inside the target.

- Primary collimator: The primary port collimator consists of a tungsten alloy block which has a density of $18 \mathrm{~g} / \mathrm{cm}^{3}$. Basically it is a cylinder with cylindrical holes drilled into it. Located directly beneath the target, its height is about $10.1 \mathrm{~cm}$, and the outer diameter is about $6.28 \mathrm{~cm}$.

- Flattening filter: Made of mild steel, attached to the lower end of the primary collimator. It is conically shaped and ends in a filter back flat and a tube surround. Because the spectral distribution of the Bremsstrahlung has an angular dependence, the dose distribution has a strong peak at the central axis. The flattening filter to achieve a flat dose profile in a tank of water under specific reference conditions [22].

- Ion chamber: The ion chamber assembly consists of a ceramic motherboard and a number of signal and polarising plates, separated by spacers made of aluminium alloy or ceramic. All plates are made from $0.012 \mathrm{~mm}$-thick Mylar film. All spacers motherboard have a $90 \mathrm{~mm}$ diameter hole and $2.7 \mathrm{~g} / \mathrm{cm}^{3}$ of the density.

- Mylar Mirror: placed on the beam central axis under the dose monitor, to enable patient set-up, and to show the position of the radiation beam.

- MLC: The MLC is used to collimate the additional beam to be able to create different complex shapes other than rectangles and modulate the intensity of 
the beam during processing. It consists of several thin rectangular plaques of tungsten alloy and has a tenonmortar shape ("Tongue and groove") along the $\mathrm{X}$ axis and a rounded end along the $\mathrm{Y}$ axis. Each plaque is called a leaf and can slide in and out of the field with an accuracy of 1 to $2 \mathrm{~mm}$. The width of the leaves is generally given as the width projected to the isocenter.

- Jaws: Jaws are used for field shaping. The pair of jaws was made of tungsten. Orthogonal adjustable jaws positioned after the MLC define the square field size usually over a range of $40 \mathrm{~cm}$ to less than $3 \mathrm{~cm}$; generally, these jaws are fixed for the time the beam is on.

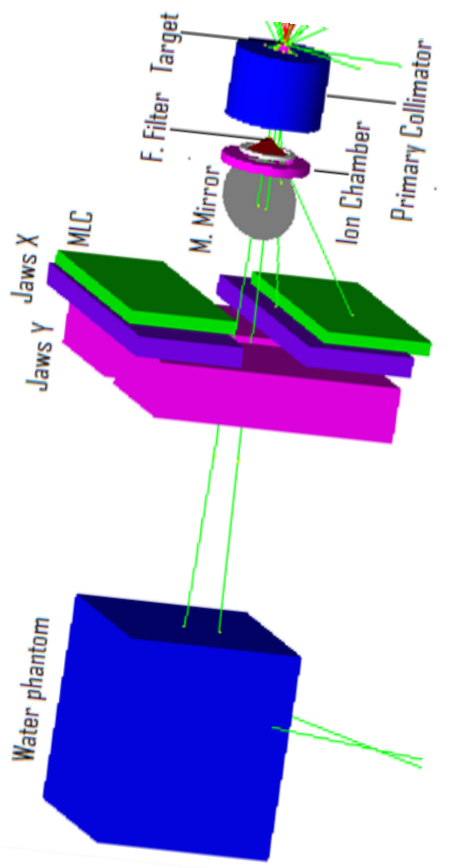

Fig. 1. Structure of the linear accelerator gantry for the $6 \mathrm{MV}$ and the simulated water phantom. All the components are not to scale.

\section{B. Grid computing methodology}

A $30 \times 30 \times 30 \mathrm{~cm}^{3}$ water phantom was simulated under the gantry with a source-surface distance $(\mathrm{SSD})$ of $100 \mathrm{~cm}$. The phantom was divided into $3 \times 3 \times 3 \mathrm{~mm}^{3}$ voxels collecting deposited energy in the phantom. A total of $2510^{9}$ of primary electrons were considered in this simulation. All calculations were performed in grid-environment using "Geant4" virtual organization (VO) [9] with a grid certificate from our national certification authorities MaGrid [10].

Our simulation runs in three steps as shown in Fig. 2. We first developed a manager which splits our simulation to a 1000 of independent jobs. Each one represents a task of 25 million of events to be run on a computing element (CE) of the Geant4 VO.

A job creates and save on the Geant4 VO storage elements (SE) about $26 \mathrm{MB}$ read out geometry (ROG) output file scoring the cumulative dose in the $3 \mathrm{D}$ voxelized water phantom.

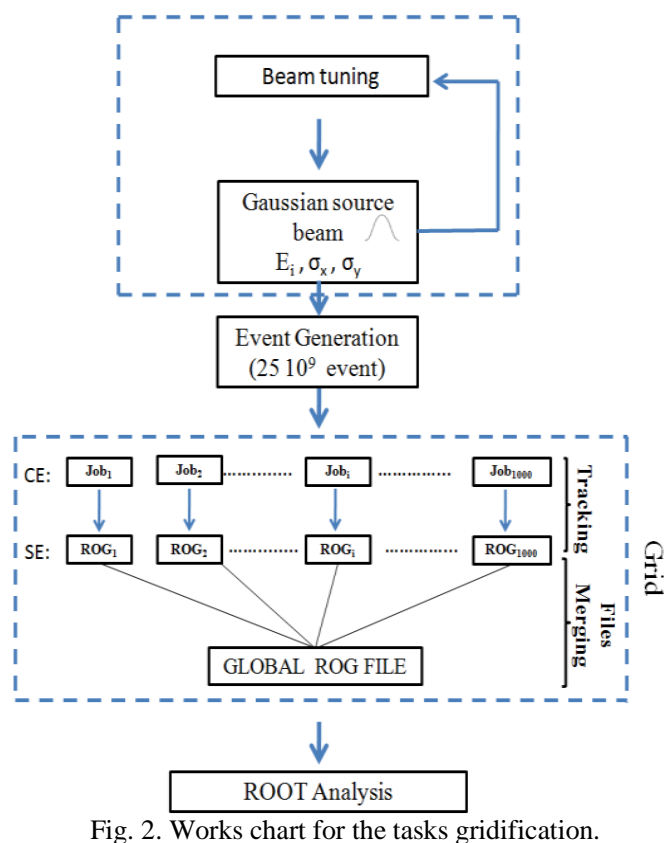

After all jobs have finished processing of their respective ROG files, merging the resulting outputs from storage elements is done in the second step by a second manger which produce a global dose file of about $36 \mathrm{MB}$. This latter is explored with ROOT in the last step to produce different dose profiles [11]-[13].

Thanks to the grid computing, the whole simulation last approximately 12 hours including the time spent in the retry of failed jobs and the results transfer from the grid to our local computing facilities. More than $95 \%$ of this allotted time was spent in the processing of the first step. Where Geant4 processes the complex particles tracking that is highly CPU time consuming. Such simulation would take more than a year on a normal processor unit.

\section{Measurement data}

The Experimental dose measurements have been obtained with an Elekta Synergy Platform linac radiotherapy unit provided by our Regional Oncology Center. The facility comprises an Elekta linac irradiation head focusing a $6 \mathrm{MV}$ photon beam onto a cube-shaped water tank (Water phantom Periphal, Model 9750 of Multidata Systems, $50 \times 50 \times 50 \mathrm{~cm}^{3}$ ) irradiated with the standard field sizes $10 \times 10 \mathrm{~cm}^{2}$.

All the measurements were obtained at a source-surface distance (SSD) of $100 \mathrm{~cm}$. A cylindrical ionization chamber of type 9732-2 Thimble having an active volume of 0.125 $\mathrm{cm}^{3}$ was used to measure the Depth and profile dose in a water phantom.

\section{Electron source}

In order to reduce the discrepancies between simulation results and measured data. The primary electron beam characteristics, namely its spot size and its mean energy, must be settled with a maximum of care [14]. The mean energy influences both the depth-dose curve and the shape of the dose profiles, while the spot size has only an impact on the dose profiles [15]. In this work, these parameters were adjusted according to the method described in [16][18]. A mean electron beam energy of $6.7 \mathrm{Me}, 3 \%$ FWHM of the mean energy and a full width at half-maximum 
(FWHM) electron spot of $3 \mathrm{~mm}$ have been found to be in good agreement with the measurements.

\section{RESULTS AND DISCUSSION}

To validate the grid model developed here. Accuracy of the simulation is evaluated by the dose error estimator [19], [20].

$$
\varepsilon=\frac{1}{N} \sum_{i} \frac{\mid \text { Dcal }_{i}-\text { Dmeas }_{i} \mid}{\text { Dmeas }_{i}},
$$

$\mathrm{i}$ corresponds to a curve point index, $\mathrm{N}$ is the number of points, Dcal ${ }_{i}$ is the dose computed at point $\mathrm{i}$ and Dmeas $\mathrm{s}_{\mathrm{i}}$ is the dose measured at point $\mathrm{i}$.

Before to present the results obtained with the reference field of $10 \times 10 \mathrm{~cm}^{2}$ we first check the tuning of the primary electron beam.

Table I compares the simulated and measured results for three beam quality parameters, the $\mathrm{TPR}_{10 / 20}$, the dose delivered at $10 \mathrm{~cm}$ depth and the position of the maximum delivered dose $\left(\mathrm{d}_{\max }\right)$. The TPR values were derived from the empirical approximation [21]:

$$
T P R_{20,10}=1.2661 D_{20,10}-0.0595
$$

Where $\mathrm{D}_{20,10}$ is the ratio of percent doses at $20 \mathrm{~cm}$ and $10 \mathrm{~cm}$ depths.

Less than $1.23 \%$ of discrepancy is observed between the simulated and the measured parameters. This shows that the

\begin{tabular}{|c|c|c|c|}
\hline & Measured & Simulated & Error $(\%)$ \\
\hline $\mathrm{TPR}_{20 / 10}$ & 0.685 & 0.684 & 0.15 \\
\hline $\mathrm{D} 10(\%)$ & 67.31 & 66.49 & 1.22 \\
\hline $\operatorname{dmax}(\mathrm{cm})$ & 1.5 & 1.5 & 0 \\
\hline
\end{tabular}
tuning of the photon beam was adjusted properly.

Let us examine the results of the simulations for dose profiles obtained with the $6 \mathrm{MV}$ photon beam and the references field.

Geant 4 and measured depth dose profiles are presented in Fig. 3. Depth dose curve were normalised to their maximum value at dmax. The simulated distribution is in good agreements with the measured one for both the build-up region $(\mathrm{Z}<\mathrm{dmax})$ and for the tail $(\mathrm{Z}>\mathrm{dmax})$ and the whole agreements is within $0.93 \%$.

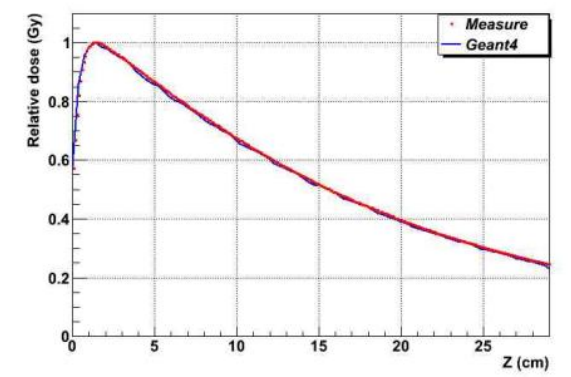

Fig. 3. Simulated and measured pdd profiles for $6 \mathrm{MV}$ photon beam using the $10 \times 10 \mathrm{~cm}^{2}$ field. The blue lines refer to Geant 4 Monte Carlo result while red dots refer to measured data.
In Fig. 4 are drawn the lateral dose profiles obtained at 1.5 $\mathrm{cm}, 5 \mathrm{~cm}, 10 \mathrm{~cm}$ and $20 \mathrm{~cm}$ depths. Simulated lateral dose profiles were normalized to the mean values in the flat zone while the measured ones were normalised to the dose along the beam axis.

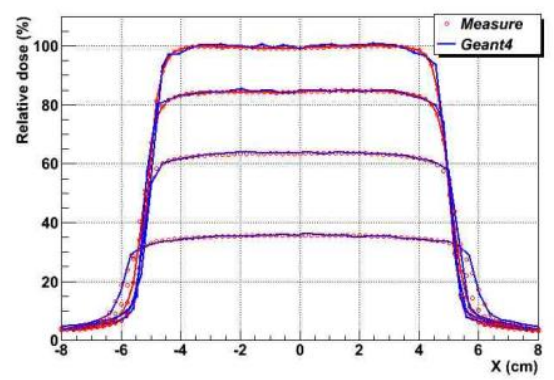

Fig. 4. Lateral dose profiles at 1.5, 5, 10 and $20 \mathrm{~cm}$ depths for $10 \times 10 \mathrm{~cm}^{2}$ field, with $6 \mathrm{MV}$ photon beam. The blue lines refer to Geant 4 Monte Carlo results; red dots refer to measured data.

For all depths, the accuracy is better than $2.63 \%$ and details of the simulations and measurements compatibility are summarized in Table II.

From all the results presented above, for the $6 \mathrm{MV}$ photon beam and a $10 \times 10 \mathrm{~cm}^{2}$ field it is easy to see that we reached a reliable level of reproducing the experimental data. Furthermore, the processing time of the simulation on the grid facilities were brought to quite reasonable duration. Therefore, this grid Geant 4 model could be applied in clinical routines as an alternative for the usual treatment planning system whenever a high accuracy is required.

TABLE II: ERRORS FOR LATERAL DOSE PROFILES AT 1.5, 5, 10 AND 20 CM OF DEPTHS FOR 6 MV PHOTON BEAM AND 10X10 $\mathrm{CM}^{2}$ FIELD.

\begin{tabular}{cc}
\hline \hline Depth $(\mathrm{cm})$ & Error $(\%)$ \\
\hline 1.5 & 1.63 \\
5 & 1.62 \\
10 & 1.86 \\
20 & 2.63 \\
\hline \hline
\end{tabular}

\section{CONCLUSIONS}

We successfully simulated an Elekta Synergy Platform medical linear accelerator using the Monte Carlo Geant4 platform and the grid computing facilities for a $6 \mathrm{MV}$ photon source and $10 \times 10 \mathrm{~cm}^{2}$ opening filed. A total of $2510^{9}$ events were processed in about 12 hours and excellent agreement were obtained with measured data for both PDD and lateral dose profiles with primary Gaussian electron beam of $6.7 \mathrm{MeV}$ mean energy and $3 \mathrm{~mm}$ FWHM width.

For all the results obtained, for the $6 \mathrm{MV}$ photon beam, it is easy to see that we have reached a reliable level of reproduction of the experimental data with an error of less than $3 \%$. In addition, the processing time of the simulation on the computing grid has been reduced to a fairly reasonable time.

This study demonstrates the possible use of Geant 4 grid simulations in radiation treatment planning. Advanced validation studies with different treatments fields and different energies are required to fully validate the accuracy of our grid model. 


\section{ACKNOWLEDGEMENTS}

This project is funded by the National Center for Scientific and Technical Research CNRST (URAC07), the High Energy Physics Cluster (RUPHE) and the SpanishMoroccans PCI2012 (A1/035250/11). The authors would like to thank the Geant 4 collaboration for providing the toolkit and examples, regular updates, documentation, and the online user forum. The authors would like also to thank Prof. Gorge Dietmar for useful discussions and Elekta's research director Kevin Brown for the given information.

\section{REFERENCES}

[1] A. Dotti, 2012, Validation of Geant4 Releases with distributed resources. Journal of Physics: Conference Series 396032033.

[2] J. Apostolakis et al, 2009. Geometry and physics of the Geant4 toolkit for high and medium energy applications Workshop on Use of Monte Carlo Techniques for Design and Analysis of Radiation Detectors, Radiat. Phys. Chem.78 859-73.

[3] Geant4 User's Guide for Application Developers by Geant4 Collaboration, Version: geant4 10.3, Publication date 9 December 2016.

[4] Vishwanath P. Singh, M.E. Medhat, N.M. Badiger, Photon energy absorption coefficients for nuclear track detectors using Geant4 Monte Carlo simulation. Radiation Physics and Chemistry, Volume 106, January 2015, Pages 83-87.

[5] M.S. Teixeira and al. da Rosa. Monte Carlo simulation of Novalis Classic $6 \mathrm{MV}$ accelerator using phasespace generation in GATE/Geant4 code. Progress in Nuclear Energy, Volume 110 January, 2019, Pages 142-147. https://doi.org/10.1016/j.pnucene.2018.09.004

[6] S. Agostinelli and al. 2003. "Geant4 a simulation toolkit". Nuclear Instruments and Methods in Physics Research Section A: Accelerators, Spectrometers, Detectors and Associated Equipment 506 (3): 250

[7] Geant4-Collaboration 2009 Physics Reference Manual for Geant4 CERN: http://www.geant4.org/geant4/support/index.shtml.

[8] Andreas Schälicke et al, 2011: Geant4 electromagnetic physics for the LHC and other HEP applications: Journal of Physics.: Conf. Ser. 331032029 doi:10.1088/1742-6596/331/3/032029.
[9] Geant4 Virtual Organization, https://lcgvoms.cern.ch:8443/vo /geant4/vomrs.

[10] Morocco Grid Computing, http://magrid.ma/.

[11] $\mathrm{Ph}$ Canal et al, 2010, Support for significant evolutions of the user data model in ROOT files, J. Phys.: Conf. Ser. 219032004 doi:10.1088/1742-6596/219/3/032004

[12] R. Brun and F. Rademakers, "ROOT-an object oriented data analysis framework," in Proceedings of the AIHENP Workshop on Nuclear Instruments and Methods in Physics Research A, pp. 81-86, Lausanne, Switzerland, September 1996.

[13] I. Antcheva et al., ROOT A C++ framework for petabyte data storage, statistical analysis and visualization, Computer Physics Communications, Volume 180, Issue 12, December 2009, Pages 2499-2512.

[14] Chetty, I.J et al., 2007. Report of the AAPM task group No. 105: Issues associated with clinical implementation of Monte Carlobasedphoton and electron external beam treatment planning. Med. Phys. 34, 4818-4853.

[15] Edwin Sham, Jan Seuntjens, Slobodan Devic, Ervin B Podgorsak, Influence of focal spot on characteristics of very small diameter radiosurgical beams, Med. Phys. 35, $3317 \quad$ (2008); http://dx.doi.org/10.1118/1.2936335.

[16] Verhaegen F and Seuntjens J, 2003. Monte Carlo modelling of external radiotherapy photon beams, Phys. Med.Biol.48R107-64.

[17] Grevillot. L et al. D, 2011. Simulation of a 6 MV Elekta Precise Linac photon beam using GATE/GEANT4, Phys. Med. Biol. 56903.

[18] S.Didi, A.Moussa, Y. Tayalati, M. Zerfaoui, "Simulation of the $6 \mathrm{MV}$ Elekta Synergy Platform linac photon beam using Geant4 Application for Tomographic Emission", Journal of Medical Physics. 2015; 40(3):136-143. doi:10.4103/0971-6203.165077.

[19] Eadi W.T. et al., Statistical methods in experimental physics (NorthHolland, Amsterdam, 1971)269.

[20] Followill DS, Taior RC, Tello VM, Hanson WF. An empirical relationship for determining photon beam quality in TG-21 from a ratio of percent depth doses. Med Phys. 1998;25(7 Pt 1):1202-05.

[21] Bak J.et al,2012. Modified dose difference method for comparing dose distributions, Journal of Applied Clinical Medical Physics, Vol. 13, No. 2, Spring 2012.

[22] F.M. Khan. The physics of radiation therapy. 2003.

[23] M. Zerfaoui, A. Rrhioua, A. Moussa, S. Didi, Y. Tayalati, M. Hamal. "GEANT4 Simulation of 192Ir Source to Study Voxelization and Number of Event Effect on the Dose Distribution", Lecture Notes in Electrical Engineering, vol 381-2016. Springer, Cham. 